\title{
Metabolic regulation by prostaglandin E 2 impairs lung group 2 innate lymphoid cell responses
}

\author{
Calum T. Robb ${ }^{1}$, You Zhou ${ }^{2}$, Jennifer M. Felton ${ }^{1}$, Birong Zhang ${ }^{2}$, Marie Goepp ${ }^{1}$, Privjyot \\ Jheeta $^{1}$, Danielle J. Smyth ${ }^{3}$, Richard M. Breyer ${ }^{4}$, Shuh Narumiya ${ }^{5}$, Henry J. McSorley ${ }^{3}$, \\ Rick Maizels ${ }^{6}$, Jürgen Schwarze ${ }^{1}$, Adriano G. Rossi ${ }^{1}$, and Chengcan Yao ${ }^{1}$ \\ ${ }^{1}$ The University of Edinburgh Centre for Inflammation Research \\ ${ }^{2}$ Cardiff University Cardiff Division of Infection and Immunity \\ ${ }^{3}$ University of Dundee Division of Cell Signalling and Immunology \\ ${ }^{4}$ Vanderbilt University Medical Center \\ ${ }^{5}$ Kyoto Daigaku Daigakuin Igaku Kenkyuka Medical Innovation Center \\ ${ }^{6}$ University of Glasgow Wellcome Centre for Molecular Parasitology
}

December 28, 2021

\begin{abstract}
Background: Group 2 innate lymphoid cells (ILC2s) play a critical role in asthma pathogenesis. Non-steroidal antiinflammatory drug (NSAID)-exacerbated respiratory disease (NERD) is associated with reduced signaling via EP2, a receptor for prostaglandin $\mathrm{E}_{2}\left(\mathrm{PGE}_{2}\right)$. However, the respective roles for the PGE 2 receptors EP2 and EP4 (both share same downstream signaling) in the regulation of lung ILC2 responses has yet been deciphered. Methods: The roles of PGE 2 receptors EP2 and EP4 on ILC2-mediated lung inflammation were investigated using genetically modified mouse lines and pharmacological approaches in IL-33- and Alternaria alternata (A.A.)-induced lung allergy models. The effects of PGE 2 receptors and downstream signals on ILC2 metabolic activation and effector function were examined using in vitro cell cultures. Results: Deficiency of EP2 rather than EP4 augments IL-33-induced lung ILC2 responses and eosinophilic inflammation in vivo. In contrast, exogenous agonism of EP4 but not EP2 markedly restricts IL-33- and Alternaria alternata-induced lung ILC2 responses and eosinophilic inflammation. Mechanistically, PGE 2 directly suppresses IL-33-dependent ILC2 activation through the EP2/EP4-cAMP pathway, which downregulates STAT5 and MYC pathway gene expression and ILC2 energy metabolism. Blocking glycolysis diminishes IL-33-dependent ILC2 responses in mice lacking endogenous PG synthesis but not in PG-competent mice. Conclusion: We have defined a mechanism for optimal suppression of lung ILC2 responses by endogenous PGE ${ }_{2}$-EP2 signaling which underpins the clinical findings of defective EP2 signaling in patients with NERD. Our findings also indicate that exogenously targeting the PGE ${ }_{2}$-EP4-cAMP and energy metabolic pathways may provide novel opportunities for treating ILC2-initiated lung inflammation in asthma and NERD.
\end{abstract}

\section{Hosted file}

Robb et al_Manuscript.docx available at https://authorea.com/users/452901/articles/550872metabolic-regulation-by-prostaglandin-e-2-impairs-lung-group-2-innate-lymphoid-cellresponses 